\title{
CARACTERIZACIÓN DE LAS COMUNIDADES VEGETALES DE LA RESERVA NATURAL RINCÓN DE SANTA MARÍA (ITUZAINGÓ, CTES) Y EL IMPACTO DE LA REPRESA HIDROELÉCTRICA YACYRETÁ SOBRE ELLAS.
}

Montiel, M. del R.; M.G. Gatti²; J.L. Fontana ${ }^{3}$; I. Zanone'; S.C. Zaninovich ${ }^{5}$

\section{RESUMEN}

La Reserva Natural Rincón de Santa María (RNRSM) fue creada con el objeto de compensar la pérdida de ambientes naturales ocasionada por el llenado del embalse al construir la Represa Yacyretá. Durante muchos años, las distintas actividades humanas dentro del área de la Reserva ocasionaron que las distintas comunidades vegetales originales cambiaran continuamente de aspecto y estructura, e incluso desaparecieran en algunos sectores al quedar submersos por el aumento del volumen de agua retenido por la presa. Actualmente, se encuentran pajonales y espartillares que están siendo afectados por invasiones de especies exóticas, y algunos individuos agrupados de especies arbóreas. Sin embargo, los bosques de ribera que existían, se han perdido completamente. En este trabajo se estudiaron las características de las comuni- dades vegetales presentes en la RNRSM, su ubicación y estado actual, con el fin de responder al interrogante sobre cómo debería ser el ecosistema natural de la Reserva, si no hubiera sufrido perturbaciones antrópicas, para así poder implementar planes de manejo y Restauración. Además, se cuantificó la pérdida de superficie de la Reserva, correlacionada con el aumento del embalse. Se partió de la hipótesis de que antes de la influencia humana, en la RNRSM existía un mosaico de vegetación formado por bosques, con características transicionales entre el bosque mesófilo y el higrófilo, junto con pajonales y espartillares. Hasta el año 2011, sobre el límite NE de la reserva, se podían encontrar isletas de bosque (11 ha). Sin embargo, luego del llenado definitivo del embalse Yacyretá en ese año, estos relictos se perdieron en su totalidad. Desde el año 2004 a la fecha, se perdieron 1421,69 ha. En la actualidad, la RNRSM presenta

\footnotetext{
1,4,5 Lic. en Ciencias Biológicas.

${ }^{2,3}$ Dr. En Biología.

1,3 Grupo Ecología y Restauración de la UNNE. Laboratorio 8 de Ecología Vegetal; Facultad de Ciencias Exactas y Naturales y Agrimensura; Av. Libertad 5460. Cel: +54 0379 154399527. Correo Electrónico: mdrmontielg@gmail.com

2,5 Instituto de Biología Subtropical

${ }^{4}$ Laboratorio de Biología de los Artrópodos. Facultad de Ciencias Exactas y Naturales y Agrimensura. Av. Libertad 5460, Corrientes, Argentina. Cel: +54 0379154934481.
} 
una predominancia de Pajonales de $A n-$ dropogon lateralis y $A$. virgatus, seguidos de Plantaciones abandonadas de $P$. elliottii, Espartillares de Elyonurus muticus y Plantaciones abandonadas de Eucalyptus sp.

\section{INTRODUCCIÓN}

En la RNRSM, se protegen comunidades vegetales seminaturales, con espartillares y pajonales de paja colorada. Fue creada junto con la Unidad de Conservación Galarza y la Reserva Natural Isla Apipé Grande en 1994 con el objeto de compensar la pérdida de ambientes naturales ocasionada por el llenado del embalse al construir la Represa de Yacyretá (Fontana et al, 2007; Fontana, 2015).

Hace aproximadamente 400 años, los pobladores locales realizaban un manejo basado en la cría ganadera con uso de fuego y extracción de madera y leña. Posteriormente, en el siglo XX, se sumó el cultivo de arroz. Unos años antes de que el área fuera declarada Reserva Natural, se establecieron plantaciones forestales con especies exóticas, como Pinus elliottii y Eucalyptus sp. Estas actividades, sumadas a la inundación permanente para el llenado del embalse de Yacyretá, ocasionaron que el ecosistema original de transición entre un bosque higrófilo y mesófilo con pajonales y espartillares, cambiara su aspecto y estructura, y en algunas partes desapareciera, al quedar submerso bajo el agua del embalse.

En este trabajo se planteó describir y georreferenciar las distintas formaciones vegetales y cuantificar los bosques, plantaciones, pajonales y espartillares de la reserva perdidos a lo largo de los últimos años debido a la elevación de la cota del embalse de Yacyretá.

\section{Ecosistemas presentes}

La Reserva se encuentra en una zona de transición entre la Provincia Paranaense, donde alternan selvas higrófilas, bosques xerófilos, esteros, lagunas y selvas marginales en galería, y el Distrito de los Campos, caracterizado por sabanas de gramíneas o pajonales altos, con isletas de bosque en galería (Cabrera, 1976). Esta región posee una larga tradición de modificaciones ambientales debido a la influencia antrópica, que se remonta a la época prehispana y se acentuó con los españoles. Con el tiempo, a las actividades tradicionales de quema, tala y ganadería se sumaron las plantaciones de arroz, cítricos y las forestaciones; modificando el ecosistema original y generando una matriz de pastizales y pajonales acompañados de isletas de bosque y cinturones de selva en galería alrededor de los cursos fluviales (Fontana, 2015). Finalmente, la construcción de la represa hidroeléctrica Yacyretá sobre el río Paraná, generó la pérdida de la selva en galería a lo largo del curso del río y de gran parte de las isletas de bosque y pajonal que habían sobrevivido a la transformación. En este contexto, se sitúa la RNRSM donde confluyen dos ecorregiones: Campos y $\mathrm{Ma}-$ lezales, y Esteros del Iberá, con influencia de las ecorregiones Delta e Islas del Paraná y Chaco Húmedo (Burkart et. al., 1999). Dentro de la reserva, y coincidiendo con el área de influencia del embalse, quedan las últimas poblaciones naturales en Corrientes de especies vegetales nativas de distribución muy restringida como Cyathea atrovirens (chachí de bañado) y Calophyllum brasiliense (arary), afectadas actualmente por la elevación de la cota del embalse. Este contexto evidencia la relevancia en términos de conservación y estudio que posee la RNRSM.

La Reserva posee las características de 
un complejo ecotono, en el que las comunidades vegetales presentan especies pertenecientes a diferentes distritos fitogeográficos y regiones naturales, a las cuales se le agregan variantes definidas por los componentes edáficos (diferentes suelos dentro de la Reserva) e hídricos (diferentes gradientes de humedad de los suelos).

Los hábitats que se han perdido en su totalidad son aquellos asociados con las selvas marginales. La presencia de especies exóticas implantadas dentro de la Reserva (Pinus elliottii y Eucalyptus sp.), si bien ejercen cambios notables en los ecosistemas de pastizales, constituyen un medio que podría propiciar la regeneración natural de las selvas marginales (Barret \& Tressens, 1996).

\section{MATERIALES Y MÉTODOS}

La RNRSM se ubica en el $\mathrm{N}$ de la provincia de Corrientes, en el Departamento de Ituzaingó, sobre el río Paraná, limitando con el embalse de Yacyretá en el N y con la ex ruta nacional No 12 hacia el S. El clima es húmedo subtropical sin estación seca. Las precipitaciones superan los $1800 \mathrm{~mm}$ anuales.

Durante los años 2015 y 2016 se realizó el reconocimiento del estado actual de los distintos ambientes de la RNRSM mediante sistema de información geográfica y recorridos a campo, durante los cuales se realizó un relevamiento de las distintas comunidades vegetales mediante la identificación visual de las especies. Aquellas que no se pudieron reconocer a campo, fueron colectadas para su determinación en herbario.

Estos datos y la observación satelital permitieron la elaboración de un mapa preliminar de vegetación. Por otro lado, se midió la pérdida de superficie de la Reserva a través del tiempo, a fin de correlacionarla con la elevación de la cota del embalse.

\section{Resultados}

Debido a las sucesivas elevaciones de la cota del embalse, la RNRSM fue perdiendo

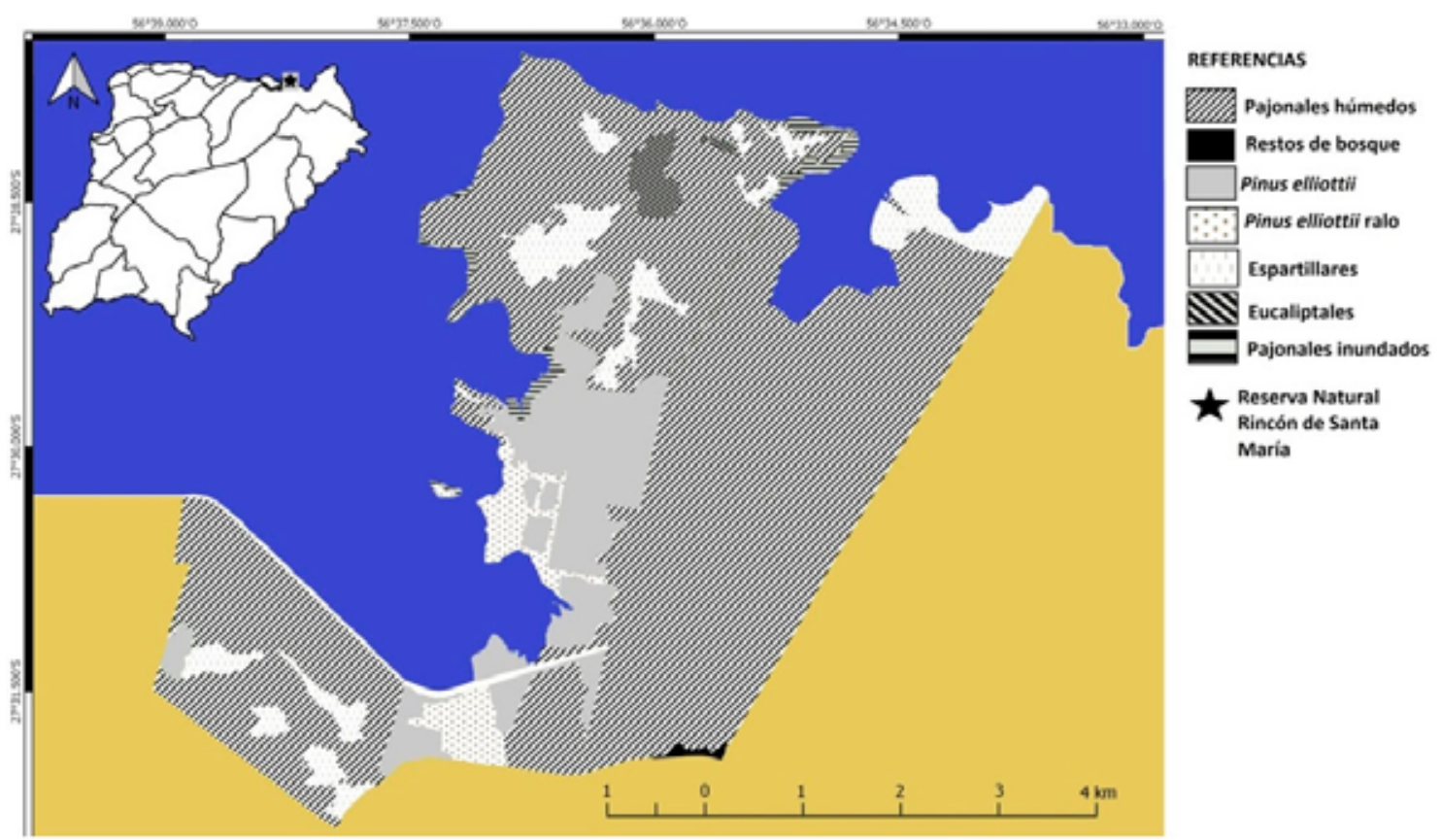

Figura 1. Comunidades vegetales presentes en la RNRSM, Corrientes, Argentina. 
su superficie considerablemente. Desde el año 2004 a la fecha, se perdieron 1421 ha. En la actualidad, la RNRSM presenta una predominancia de pajonales de Andropogon lateralis y $A$. virgatus, seguidos de plantaciones abandonadas de $P$. elliottii, espartillares de Elyonurus muticus y plantaciones abandonadas de Eucalyptus sp (Figura 1).

\section{COMUNIDADES VEGETALES}

\section{Pajonales húmedos con A. lateralis y A. virgatus}

Con un área de 2474 ha los pajonales húmedos son la comunidad vegetal dominante de la RNRSM. Cubren los suelos más húmedos y con poca capacidad de drenaje. Estos ambientes han sufrido intervenciones sucesivas (movimientos de obra, cultivos, sobrepastoreo y forestaciones) que han alterado radicalmente la composición original de las comunidades vegetales. Sumado a esto, está en riesgo su subsistencia por la alta capacidad de diseminación de los pinos que invaden su superficie. Es por ello, que en algunos sectores de estos pajonales, se encuentran pinos aislados, producto de la dispersión anemócora.

\section{Plantaciones de Pinus elliottii}

Se encuentran distribuidas en varios sitios de la RNRSM comprendiendo un área total de 419 ha. Estas plantaciones presentan distintas edades y distribución espacial. La composición específica del sotobosque de la plantaciones de P. elliottii es muy variable. En algunos sectores se puede encontrar una alta abundancia de helechos herbáceos, como Pteris denticulata. Sin embargo, en las plantaciones con mayor incidencia de radiación, el sotobosque se caracterizaba por la predominancia de gramíneas. En cuanto a la vegetación arbórea nativa, se encontró dentro de las plantaciones, distintos renovales y juveniles de Balfourodendron riedelianum, Enterolobium contortisiliquum, Peltophorum dubium, Nectandra sp., Syagrus romanzzofiana, Allophyllus edulis, Cecropia pachystachya, Croton urucurana, Fagara sp., Sapium haematospermum, Acrocomia aculeata y Tabernaemontana australis.

\section{Espartillares}

Con una superficie de 274 ha, esta comunidad vegetal se distribuye en las zonas altas de la RNRSM, arenosas, no inundables. Está representada por la especie Elyonurus muticus. La falta de quema del espartillar permite la acumulación de una cantidad importante de hojarasca que impide el crecimiento de numerosas especies pequeñas, y los peligros de incendios.

\section{Plantaciones de P. elliottii ralo}

Esta comunidad vegetal está representada por pinos que se encuentran muy separados espacialmente entre sí y de manera irregular, permitiendo la entrada de luz hacia el interior, desarrollándose en su interior pajonales de $A$. lateralis y $A$. virgatus. El área total de esta comunidad vegetal es de 112 ha. Como puede observarse en el mapa, éstas se distribuyen en las cercanías de las forestaciones más densas. Debido a la quema y explotación maderera, se volvieron plantaciones con distribución irregular que permitieron el desarrollo de gramíneas y ocasionalmente especies del bosque (como Cecropia pachystachya).

\section{Pajonales inundados:}

Compuestos principalmente por Andropogon lateralis, los mismos se encuentran 
bordeando parte de la presa, ocupando un área de 42 ha. Debido al avance del agua por la subida de la cota del embalse de Yacyretá, es probable que con el tiempo, den origen a comunidades vegetales higrófilas, como cañaverales y camalotales, o que desaparezcan como consecuencia del oleaje de la presa.

\section{Eucaliptales}

Con un área de 40 ha ésta comunidad vegetal está formada principalmente por individuos de Eucalyptus sp, en distintos estadíos de crecimiento, encontrándose ejemplares de hasta $40 \mathrm{~m}$ de altura. Debajo de los mismos, crecen pajonales con Andropogon lateralis y $A$. virgatus. Además, mezclados con Eucalyptus sp, se encuentran ejemplares de Pinus elliottii.

\section{Restos de bosque}

Gracias a los esfuerzos de los guardaparques, se recuperaron 5 ha de bosque. En un primer momento, los guardaparques de la reserva ubicaron ejemplares de distintas especies nativas como Enterolobium contortisiliquum, Nectandra sp., Syagrus romanzzofiana, Cecropia pachystachya, Croton urucurana, Fagara sp., Sapium haematospermum y Tabernaemontana catharinensis que con el tiempo, dieron lugar a la llegada de otras especies nativas, formando pequeños cordones de bosque.

\section{PÉRDIDA DE AMBIENTES}

Debido a las sucesivas elevaciones de la cota del embalse de Yacyretá, aquellos ambientes ubicados en la rivera de la presa de la RNRSM se vieron afectados. Por medio de imágenes satelitales, se contabilizaron éstos cambios. La figura 2 resume estas pérdidas.

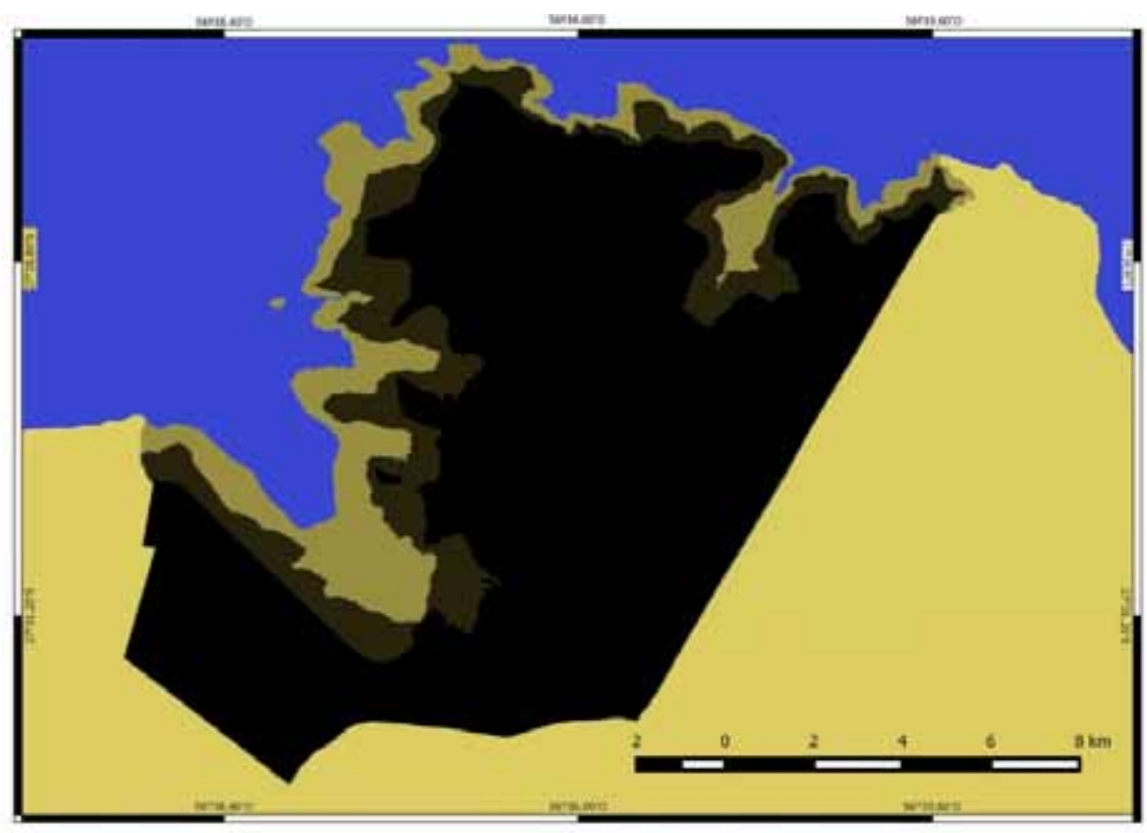

MEFERENCIAS

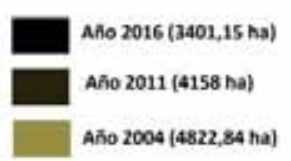

Figura 2. Comunidades vegetales presentes en la RNRSM 
En el año 2004, la RNRSM contaba con 4822 ha (Figura 3), de las cuales 11 ha eran de bosque ribereño, ubicado en el borde NE de la Reserva. En el año 2011, la Reserva contaba con 4158 ha, habiendo perdido 665 ha durante este período. Con la elevación final de la cota del embalse durante ese año, la RNRSM quedó 3401 ha, un total de 757 ha menos que en el año 2011, y 1422 ha menos que en 2004.

\begin{tabular}{|c|c|c|c|}
\hline Año & Área (ha) & Pérdida (ha) & \\
\hline 2004 & 4822 & & \\
\hline 2011 & 4158 & 665 & \\
\hline 2016 & 3401 & 757 & 1422 \\
\hline
\end{tabular}

Figura 3. Pérdida de superficie de la RNRSM

DIAGNÓSTICO DEL ESTADO ACTUAL DE LA VEGETACIÓN

El uso ganadero prolongado de los campos correntinos ha modificado la composición florística y estructural de los mismos. Eskuche \& Fontana (1996) señalaba que los pastizales del $\mathrm{NE}$ de Corrientes y $\mathrm{S}$ de $\mathrm{Mi}$ siones, de Andropogon lateralis, los flechillares de Aristida jubata y los espartillares de Elionurus muticus evolucionaban hacia praderas de Axonopus compressus y Paspalum notatum por acción de las quemas y el pastoreo.

Las formaciones boscosas han sufrido un fuerte impacto. Los bosques mesófilos están asentados sobre suelos con aptitud agrícola y debido a ello en numerosos casos han sido sometidos a un fuerte proceso de degradación, mientras que otros han sido destruidos.

La zona donde se encuentra la Reserva fue especialmente sometida a una serie de intervenciones antrópicas, tales como plantaciones de arroz en los sectores bajos y plantaciones de soja en las lomadas no alcanzadas por inundaciones, además del uso ganadero y forestal. Posteriormente, se sumó la remoción de suelo, el movimiento de maquinarias y la construcción de la presa lateral izquierda y del embalse Yacyretá, que atraviesa en forma oblicua a la Reserva, lo cual influyó sobre la composición y distribución florística original.

\section{Flora exótica}

Tal como se describió con anterioridad esta reserva presenta plantaciones de Eucalyptus sp. y Pinus elliottii de al menos 40 años de edad.

Respecto de Eucalyptus sp. existen antecedentes en el hemisferio $\mathrm{N}$ sobre los cambios desfavorables en el ambiente que produce su plantación, ya que acidifican el suelo (Vázquez-Yanes \& Batis, 1996). Se ha observado frecuentemente en el NO y $\mathrm{N}$ de la provincia de Corrientes, y específicamente en la RNRSM, la regeneración natural de especies leñosas dentro de las plantaciones de Eucalyptus sp. (Barret \& Tressens, 1996). Los mismos autores, y el Grupo de Investigación en Ecología y Restauración de la UNNE, han observado que el sotobosque de estas plantaciones está compuesto por especies nativas, mayoritariamente provenientes de bosques higrófilos vecinos, por lo que en caso de querer iniciar tareas de restauración de bosques, las 
plantaciones de Eucalyptus sp. ayudarían a crear condiciones ambientales convenientes para que las especies del bosque puedan recuperarse (Barret \& Tressens, 1996).

\section{DISCUSIÓN Y CONCLUSIONES}

En la actualidad, la RNRSM presenta una predominancia de pajonales de Andropogon lateralis y $A$. virgatus, y superficies menores de plantaciones abandonadas de $P$. elliottii, espartillares de Elyonurus muticus y plantaciones de $E u-$ calyptus sp. Presenta una pequeña porción (5 ha) de bosque, que a pesar de estar formado por especies nativas, fue implantado por los Guardaparques de la Reserva.

Dentro de las plantaciones de especies exóticas, se observó la regeneración natural de algunas especies nativas, como Cecropia pachystachya, Sapium haematospermum, Acrocomia aculeata, Tabernaemontana catharinensis y Fagara sp., cuyas semillas llegarían por dispersión desde relictos de bosque cercanos a la Reserva. Estudios realizados en el NO Correntino (Barrett \& Tressens, 1996) sobre plantaciones de Eucalyptus sp. demuestran el potencial de esta especie para la regeneración arbórea nativa. Es por ello, que se recomienda un estudio exhaustivo sobre los determinantes bióticos y abióticos que permiten el desarrollo de la vegetación arbórea nativa dentro de los Eucaliptales de la Reserva, para formular futuros planes de Restauración acordes a la región. Asimis- mo, debido a la abundancia que presenta la especie Pinus elliottii dentro de la RNRSM, y a que no se puede controlar su dispersión sobre los pastizales, debe ser considerada la opción de removerlos de la Reserva.

Como se expuso en este trabajo, la Represa Hidroeléctrica de Yacyretá ejerce una importante presión sobre los ambientes de la RNRSM: Está llevando a la desaparición y disminución de muchos de ellos, con las consecuencias que esto acarrea. Se recomienda proyectos de acción que eviten la continua erosión por oleaje que produce la disminución del territorio de la Reserva, en los límites de la presa. En el período comprendido entre los años 2009-2012, el Grupo Ecología y Restauración de la UNNE liderado por el Dr. José Luis Fontana, llevó a cabo un estudio de Restauración experimental de una parcela con elementos de la selva riparia y del bosque mesófilo, en un sitio elegido de la RNRSM. Los trabajos de restauración se hicieron respetando los procesos sucesionales. Estudios fitosociológicos previos permitieron conocer las comunidades vegetales y las distintas etapas de sucesión, esencial en cualquier proyecto de restauración de ecosistemas. Mediante este estudio, se logró establecer parcelas de restauración para rescatar a la especie arbórea Calophyllum brasiliense y al helecho arborescente Cyathea atrovirens. Dicho proyecto fue interrumpido por la no renovación del convenio de trabajo con la Entidad Binacional Yacyretá. 


\section{BIBLIOGRAFÍA}

BARRET, W. \& S.G. TRESSENS. 1996. Estudio de la Vegetación Nativa en Plantaciones de Eucalyptus grandis (Myrtaceae) en el Norte de la Provincia de Corrientes, Rep. Argentina. Bonplandia, 9 (1-2). Reeditado en SAGyA Forestal.

ESKUCHE, U.G. \& J.L. FONTANA. 1996. La vegetación de las Islas argentinas del Alto Paraná. II. Las comunidades de campo abierto. Folia Bot. et Geobot. Correntesiana 12: $3-15+2$ tablas. Corrientes.
FONTANA, J.L. 2015. ¿Por qué restaurar ecosistemas? La experiencia con los bosques ribereños del embalse Yacyretá, provincia de Corrientes". Extensionismo, Innovación y Transferencia Tecnológica - Claves para el desarrollo - Vol. 2: 148156. Fac. de Cs. Exactas y Nat. y Agrim., UNNE. Corrientes. ISSN 2422-6424.

FONTANA, J.L. 2015. Flora y Vegetación del Nordeste de Corrientes y sur de Misiones. En: El Patrimonio Natural y Cultural en el área de influencia del Embalse de Yacyretá, Argentina. Cap. 1, P. 9-27. Fundac. Felix de Azara. Buenos Aires. ISBN 978-987-3781-21-6. 